\title{
Adult Niemann-Pick disease type B with myositis ossificans: \\ a case report
}

\author{
R. Shumnalieva ${ }^{1}$, S. Monov ${ }^{1}$, V. Shoumnalieva - Ivanova², R. Rashkov ${ }^{1}$, R. Stoilov ${ }^{1}$ \\ ${ }^{1}$ Rheumatology Department, Medical University - Sofia, Bulgaria \\ 2Ophthalmology Department, Medical University - Sofia, Bulgaria
}

\section{ABSTRACT}

Niemann-Pick disease (NPD) is a rare autosomal recessive lysosomal lipid storage disorder. It is caused by mutations of genes which products are involved in the metabolism of sphingolipids. Their dysfunction causes sphingomyelin to accumulate in different organs which leads to progressive multisystemic disorder. Type A and type B NPD are caused by mutations in sphingomyelin phosphodiesterase-1 gene with deficiency of acid sphingomyelinase (ASM). Type C and type D NPD have normal or reduced sphingomyelinase activity but differ pathogenetically from type A and B. The various types share common clinical features and the severity of the disease varies depending on the gene mutation, enzyme deficiency and the system involved. The estimated incidence of type A and B NPD is 1:250000 and of type C is 1:150 000 live births.

\section{ANAMNESIS}
A 34-year old man from gypsy origin presented at the rheumatology clinic with a 6-months history of:
$\Rightarrow$ walking difficulties;
$\Rightarrow$ pain in the pelvic area;
$\Rightarrow$ pain and limited motions in the hip joints with irradiation to the right knee;
$\Rightarrow$ morning stiffness lasting more than one hour;
$\Rightarrow$ short painless periods when taking NSAIDs.

\section{PHYSICAL EXAMINATION}

$\Rightarrow$ dysmorphic face with gothic palathum;

$\Rightarrow$ chest deformity, expiratory wheezing with normal breath sounds;

$\Rightarrow$ hepatosplenomegaly - up to $3 \mathrm{~cm}$ for the liver and 8 for the splen;

$\Rightarrow$ difficulties walking, limited range of motion in the hip joints, internal rotation of the right hip joint, pain and limited range of motion in the right knee joint with a flexion contraction at $45^{\circ}$, pain and limited range of motion in the lumber spine;

$\Rightarrow$ pancerebellar syndrome, adiadochokinesis, quadripiramid syndrome, no abdominal reflexes, polyneuropathy syndrome;

$\Rightarrow$ cognitive dysfunction.

\section{PLAIN RADIOGRAPHS}
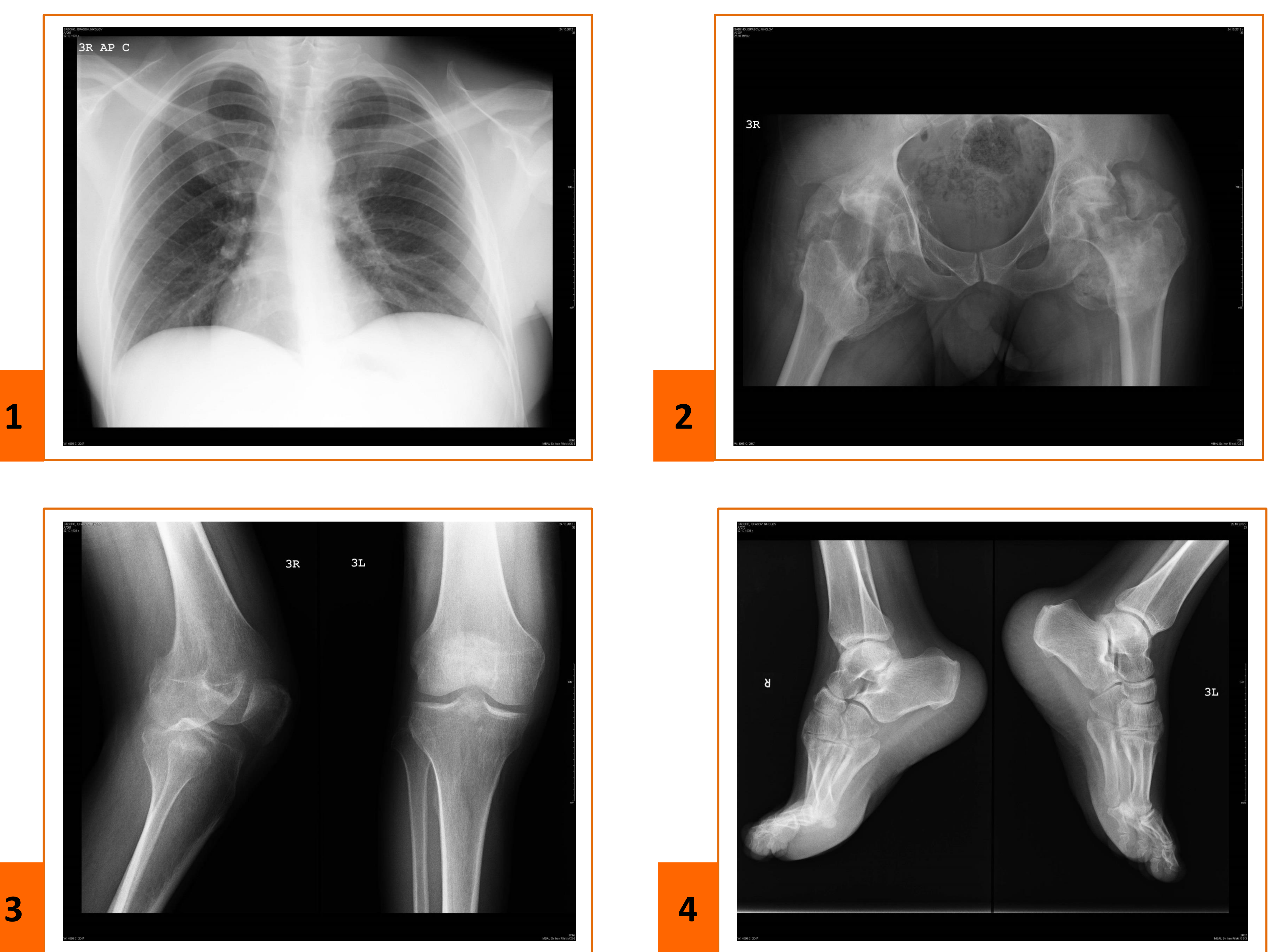

Pic. 1. Denser hilus, reticular interstitial pattern of lung involvement, without infiltrates;

Pic. 2, 3, 4. Massive ossifications with amorphous character around the hip joints; right hip joint in internal rotation with internal rotation in the knee joint, high foot arches, a heel-spur on the right site. Hip, knee and ankle joints - with normal joint spaces.
His past anamnesis and family history revealed:

$\Rightarrow$ failure to thrive, walking and talking difficulties

$\Rightarrow$ hepatosplenomegaly diagnosed at the age of 2;

$\Rightarrow$ psychomotor retardation and deterioration of neurological development;

$\Rightarrow$ diagnosis of paranoid schizophrenia at the age of 32;

$\Rightarrow$ family history of a brother who died from pneumonia, a death sister with a history of asthma, a brother with hepatosplenomegaly and a cousin with Niemann-Pick disease type B.

\section{LABORATORY DATA}

\begin{tabular}{|c|c|c|c|c|c|c|c|c|c|c|}
\hline ESR & CRP & $\mathrm{Hb}$ & RBC & WBC & \multicolumn{2}{|c|}{ PLC } & gluc & creat & uric & urea \\
\hline 18 & 1,41 & 135 & 4,95 & 3500 & 730 & & 4,19 & 56 & 260 & 5,8 \\
\hline AsAT & AIAT & CPK & GGT & AF & LDH & ASM & & \begin{tabular}{l|l} 
Prot & $a$
\end{tabular} & album $f$ & fibrino \\
\hline 34 & 1 & 1 & 1 & 164 & 266 & 2,7 & & 74 & 44 & A12 \\
\hline tBil & dBil & vit. $B_{12}$ & folat & chol & TG & HD & DL & LDL & VLDL & $\mathrm{Ca}^{2+}$ \\
\hline 8,45 & 2,8 & 495 & 7,82 & 4,87 & 1,79 & 1,0 & 03 & 2,97 & 0,77 & 2,2 \\
\hline
\end{tabular}

\section{CT IMAGES}
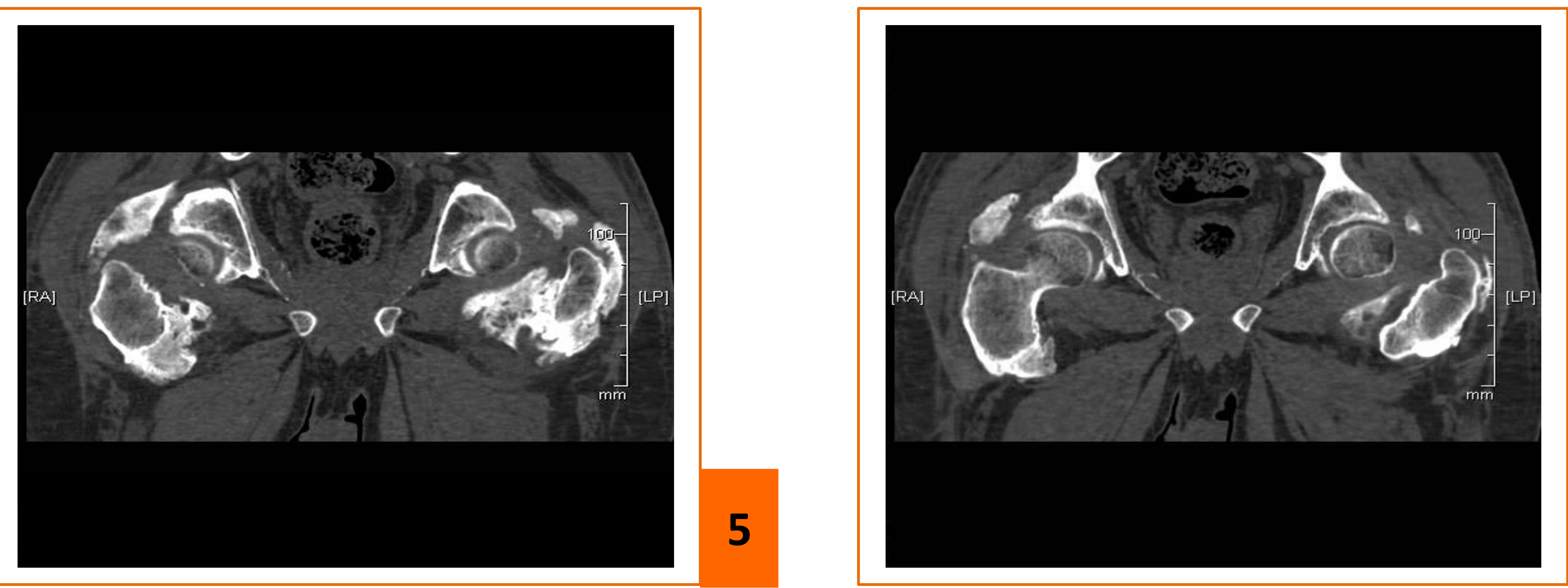

6
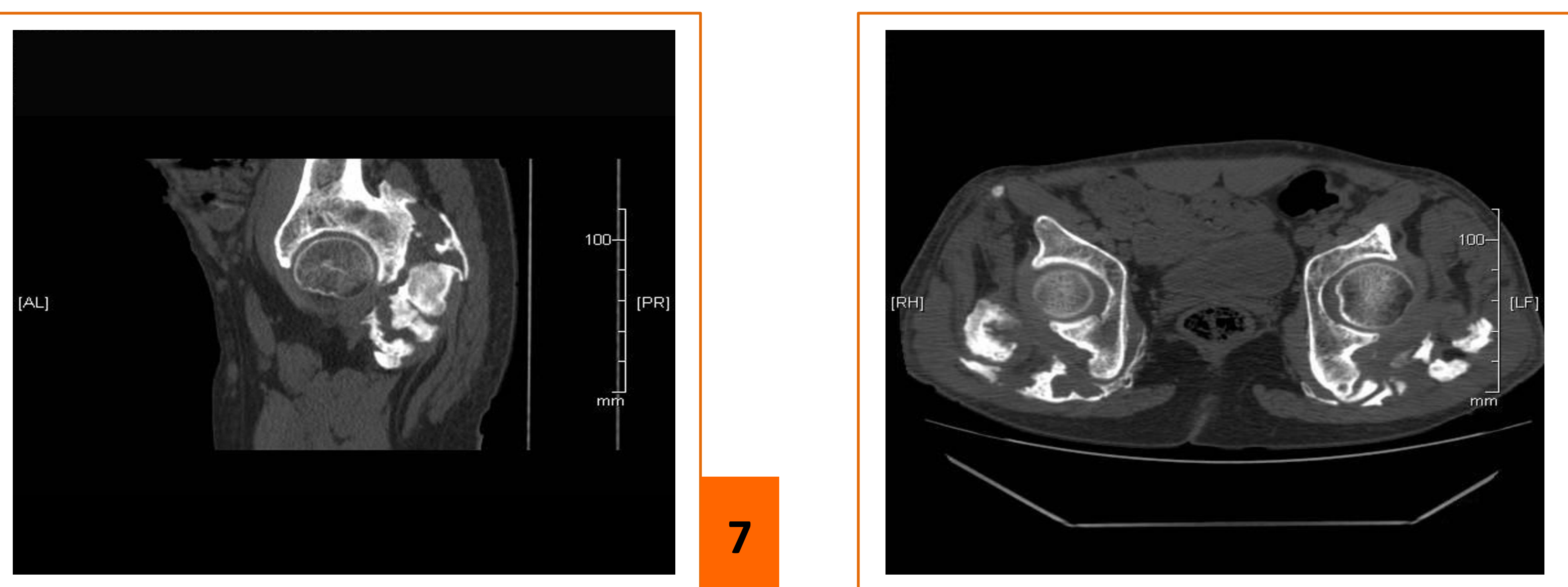

8

Pic. 5, 6, 7, 8. Multiple massive ossifications and exostoses around the iliac bones and greater trochanter bilateral, in the internal and external obtorator muscles, gluteus medius and minimus and quadratus femoris muscle.

The images were suggestive of myositis ossificans.

\section{CONCLUSION}

It is important to raise the awareness of this debilitating condition and the need of a multidisciplinary management of such patients. As there is no recognized effective treatment for this disorder the possibility for prenatal diagnosis through amniocentesis or chorionic villus sampling especially in familial cases is of great importance. 